\title{
Evaluación de minerales alumino silicatos de Norte de Santander para fabricar piezas cerámicas de gran formato
}

\author{
Norte de Santander Alumino silicates Minerals' Evaluation for the \\ Big Scale Ceramic Pieces Manufacturing
}

\section{Avaliação de minerais aluminossilicatos do Norte de Santander para fabricar peças cerâmicas de grande formato}

\section{Resumen}

Se dan a conocer los resultados de la influencia del feldespato al ser mezclado con arcilla del área metropolitana de Cúcuta (Norte de Santander, Colombia), en relación con las principales variables que son tenidas en cuenta a la hora de fabricar un producto cerámico tradicional de gran formato mediante técnica de conformado por extrusión. Los materiales empleados fueron arcillas provenientes de las formaciones León y Guayabo, y el feldespato del municipio de Sardinata, los cuales fueron estudiados mediante difracción de rayos (DRX) y fluorescencia de rayos X (FRX). Las probetas conformadas se realizaron empleando proporciones de 100:0, 90:10 y 80:20 de arcilla-feldespato, respectivamente, $\mathrm{y}$ fueron sometidas a cocción a las temperaturas de $950^{\circ} \mathrm{C}-1000^{\circ} \mathrm{C}-1050^{\circ} \mathrm{C}$. Se observó que la adición de feldespato mejoró de manera significativa las propiedades estudiadas para todas las arcillas empleadas en comparación con la mezcla patrón. Las muestras preparadas con arcilla Támesis presentaron el mejor desempeño.

Palabras clave: Alabeo, Aluminosilicatos, Arcilla Támesis, Cerámica roja, Feldespato.

\footnotetext{
* Universidad Francisco de Paula Santander (San José de Cúcuta-Norte de Santander, Colombia). sandrarozo85@hotmail.com

** M. Sc. Universidad Francisco de Paula Santander (San José de Cúcuta-Norte de Santander, Colombia). j_sanchezmolina@yahoo.es

*** Universidad Francisco de Paula Santander (San José de Cúcuta-Norte de Santander, Colombia). johngelves@yahoo.es
} 


\section{Abstract}

This study shows the influence results of adding feldspar to clay materials from the metropolitan area of Cucuta (Colombia), in relation to key variables that are taken into account, when making a large format traditional ceramic product, through technical extrusion forming. The materials used were clays from the León and Guayabo formations, and the feldspar from the Sardinata town, all of which were studied by X-ray diffraction (XRD) and X-ray fluorescence (XRF). The specimens were formed in proportions of 100:0, 90:10 and 80:20 clay-feldspar, and were subjected to baking at temperatures of $950{ }^{\circ} \mathrm{C}-1000{ }^{\circ} \mathrm{C}-1050{ }^{\circ} \mathrm{C}$. It was observed that the addition of feldspar, significantly improved the properties studied for all clays used in comparison with the standard mixture. The samples prepared with Támesis clay presented the best performance.

Keywords: Red Ceramics, Feldspar, Warping, TámesisClay.

\section{Resumo}

São apresentados os resultados da influência do feldspato ao ser misturado com argila da área metropolitana de Cúcuta (Norte de Santander, Colômbia), em relação com as principais variáveis que são levadas em consideração à hora de fabricar um produto cerâmico tradicional de grande formato mediante técnica de conformado por extrusão. Os materiais empregados foram argilas provenientes das formações León e Guayabo, e o feldspato do município de Sardinata, os quais foram estudados mediante difração de raios X (DRX) e fluorescência de raios X (FRX). As provetas conformadas se realizaram empregando proporções de 100:0, 90:10 e 80:20 de argilafeldspato, respectivamente, e foram sometidas a cocção às temperaturas de $950^{\circ} \mathrm{C}-1000^{\circ} \mathrm{C}-1050^{\circ} \mathrm{C}$. Observouse que a adição de feldspato melhorou de maneira significativa as propriedades estudadas para todas as argilas empregadas em comparação com a mistura padrão. As amostras preparadas com argila Támesis apresentaram o melhor desempenho.

Palavras chave: Empenamento, Aluminossilicatos, Argila Támesis, Cerâmica vermelha, Feldspato. 


\section{INTRODUCCIÓN}

El área metropolitana de Cúcuta (Norte de Santander, Colombia) se caracteriza por ser un gran centro de producción de materiales cerámicos de construcción; alrededor de 59 empresas legalmente constituidas se dedican allí a esta actividad. La actividad productiva de este conglomerado se fundamenta en la elaboración de artículos de cerámica roja: baldosas, tejas, ladrillos y bloques, los cuales se distribuyen tanto a nivel nacional como internacional [1].

La gran disponibilidad de materiales arcillosos en esta región ha permitido el desarrollo de esta industria durante muchos años; sin embargo, el auge de la globalización, con la fuerte competencia en mercados internacionales, y las inestabilidades políticas de los mercados tradicionales de exportación del sector han llevado a una fuerte desaceleración en la economía regional, al punto de llevar al cierre de algunas fábricas [2]. Simultáneamente, los productos cerámicos de gran formato (mayores de $33 \mathrm{~cm}$. en por los menos uno de sus lados), empleados en pisos y revestimientos, se han venido imponiendo dentro de la actividad constructiva, hecho que ha afectado la producción local, ya que gran parte de sus productos se encuentra limitada a formatos inferiores a los 33 centímetros [6].

En consecuencia, diferentes estudios realizados en relación con los aspectos competitivos del sector [3-5] han dejado ver la necesidad de innovar y diversificar la producción de los artículos cerámicos, así como mejorar algunos aspectos de los ya existentes, en especial los relacionados con tolerancias dimensionales, cuyo objetivo es dar cumplimiento a los requerimientos técnicos de los principales mercados potenciales, hecho que hasta ahora ha limitado el acceso a ellos.
El tipo de conformado (moldeo por extrusión) y las pastas empleadas (mezclas de arcillas y arena) hasta ahora en las empresas de la zona no han permitido el desarrollo satisfactorio de artículos de gran formato; los resultados obtenidos se caracterizan por la formación de alabeo, pérdidas de resistencia mecánica y un aumento significativo de la rotura en el horno [67].

Además de las arenas, los feldespatos han sido empleados con éxito en la formulación de pastas cerámicas, con especial uso para la fabricación de artículos de cerámica esmaltada, donde las temperaturas de cocción superan los $1100^{\circ} \mathrm{C}$ (temperatura promedio para la fusión de esta clase de minerales); sin embargo, su uso en la fabricación de cerámica roja tradicional no es muy común, puesto que las temperaturas de cocción de ésta es más baja que la temperatura de fusión de los feldespatos (raramente superan $\operatorname{los} 1080^{\circ} \mathrm{C}$ ), hecho que de forma ideal afectaría la formación de las fases que favorecen la resistencia mecánica de la pieza cocida, así como la de su estabilidad dimensional [8-9].

El objetivo del presente trabajo es evaluar el efecto de la adición de materiales feldespáticos a los materiales arcillosos típicos empleados en la región sobre las principales variables tenidas en cuenta para la fabricación de artículos cerámicos de gran formato, conformados por la técnica de extrusión, a fin de promover o descartar su uso en las empresas del sector cerámico regional.

\section{Materiales Y MÉTODOS}

Fueron empleadas 4 muestras arcillosas provenientes de las principales minas de la región, las cuales se presentan en la Tabla 1.

\section{TABLA 1}

\section{Muestras ARCILLOSAS EMPLEADAS EN EL PROYECTO}

\begin{tabular}{|c|c|c|c|}
\hline Mina & Abreviatura & Municipio & Formación geológica \\
\hline Alejandra & A & El Zulia & Grupo Guayabo \\
\hline Chía & C & El Zulia & Grupo Guayabo \\
\hline Támesis & T & Los Patios & Grupo Guayabo \\
\hline Peracos & P & Cúcuta & Formación León \\
\hline
\end{tabular}


El material feldespático empleado se obtuvo de la mina propiedad de la empresa Cerámica Italia S.A., ubicada en el municipio de Sardinata (Norte de Santander), cuya abreviatura empleada en este trabajo fue la letra F.

Se formularon mezclas en las proporciones 90:10 y 80:20, de arcilla y feldespato, respectivamente. De forma adicional, se preparó una muestra sin adición de este material, como elemento patrón de comparación.

Dichas pastas se molturaron hasta un pasante malla 30; posteriormente se humectaron con humedad constante del $20 \%$ para todas las muestras, para dar paso al proceso de extrusión; el secado de las probetas se llevó en dos etapas: el primero como secado natural durante 16 horas, y segundo en estufa eléctrica a $110^{\circ} \mathrm{C}$ durante 24 horas; la etapa de cocción se realizó en horno mufla en atmósfera oxidante, a las temperaturas de $950^{\circ} \mathrm{C}$, $1000^{\circ} \mathrm{C}$ y $1050^{\circ} \mathrm{C}$.

Técnicas y equipamiento empleado. Para la realización de la investigación se contó con un molino de martillos de laboratorio marca Servitech, modelo CT-058, empleado para la molturación de las pastas preparadas; una extrusora de laboratorio con vacío, modelo NEW WAVE, fabricada por la empresa Metal Souza Ltda., con molde macizo, para el conformado de probetas tipo baldosa con un ancho de salida de $4,0 \mathrm{~cm}$; una estufa de secado marca "Dies", cuyo calentamiento se hace con resistencia eléctrica; un horno mufla modelo 10/12, marca Gabrielli; una balanza digital, marca OHAUS, y un calibrador digital Mitutoyo. La medición de la resistencia a la flexión (módulo de rotura) se llevó a cabo en un equipo marca
Gabrielli, aplicando la norma técnica colombiana NTC4321-4; de igual modo, la absorción de agua se llevó a cabo siguiendo los parámetros de la norma NTC 4321-3 en un equipo artesanal construido para tal fin.

La caracterización mineralógica se realizó mediante difracción de rayos $\mathrm{X}$, por el método de polvos; el equipo empleado fue un difractómetro Rigaku D/ MAX IIIB con radiación de $\mathrm{Cu} \mathrm{K \alpha}$ en las condiciones de $30 \mathrm{Kv}$ y $15 \mathrm{~mA}$. El escaneo se llevó a cabo por el método paso a paso, con un paso de $0.02^{\circ}$ y un tiempo de conteo por paso de 2 segundos, para un rango de $2 \theta$ entre $3^{\circ}$ y $70^{\circ}$. El software de análisis empleado fue el XpertHighScore Plus de PANalytical. La composición química se llevó a cabo mediante la técnica de fluorescencia de rayos $\mathrm{X}$, empleando un equipo de la marca PANalytical modelo Axios, empleando el software de trabajo WROXI.

La caracterización fisico-cerámica hace referencia a los cambios presentados en los parámetros de contracción de secado y cocción, resistencia a la flexión y \% de absorción de agua.

\section{RESUlTADOS Y DISCUSIÓN}

\section{A. Caracterización química y mineralógica}

Los resultados de la caracterización química, mediante FRX, son mostrados en la Tabla 2, y en la Fig. 1 se presenta el resultado de la caracterización mineralógica de las materias primas empleadas.

\section{TABLA 2}

ANÁLISIS QUÍMICO DE LAS MATERIAS PRIMAS EXPRESADAS EN FORMA DE ÓXIDOS (FRX)

\begin{tabular}{|c|c|c|c|c|c|}
\hline Compuesto & Alejandra & Chía & Tamesis & Peracos & Feldespato \\
\hline $\mathbf{S i O}_{2}$ & 64,8 & 65,8 & 61,3 & 67,08 & 74,4 \\
\hline $\mathbf{A l}_{2} \mathbf{O}_{3}$ & 19,2 & 18,0 & 21,7 & 24,29 & 14,5 \\
\hline $\mathbf{F e}_{2} \mathbf{O}_{3}$ & 4,8 & 6,0 & 5,4 & 5,21 & 1,1 \\
\hline $\mathbf{T i O}$ & 0,6 & 0,9 & 0,9 & 0,88 & 0,2 \\
\hline $\mathbf{C a O}$ & 0,6 & 0,3 & 0,3 & 0,43 & 0,1 \\
\hline $\mathbf{M g O}$ & 0,6 & 0,7 & 0,8 & 0,52 & 0,2 \\
\hline $\mathbf{N a} \mathbf{O}$ & 0,2 & 0,1 & 0,3 & 0,45 & 2,5 \\
\hline $\mathbf{K}_{2} \mathbf{O}$ & 1,4 & 1,7 & 2,1 & 1,14 & 5,3 \\
\hline $\mathbf{S O}_{3}$ & 0,1 & 0,1 & 0,1 & 0,1 & 0,1 \\
\hline $\mathbf{P}_{2} \mathbf{O}_{5}$ & 0,0 & 0,1 & 0,2 & 0,1 & 0,0 \\
\hline
\end{tabular}




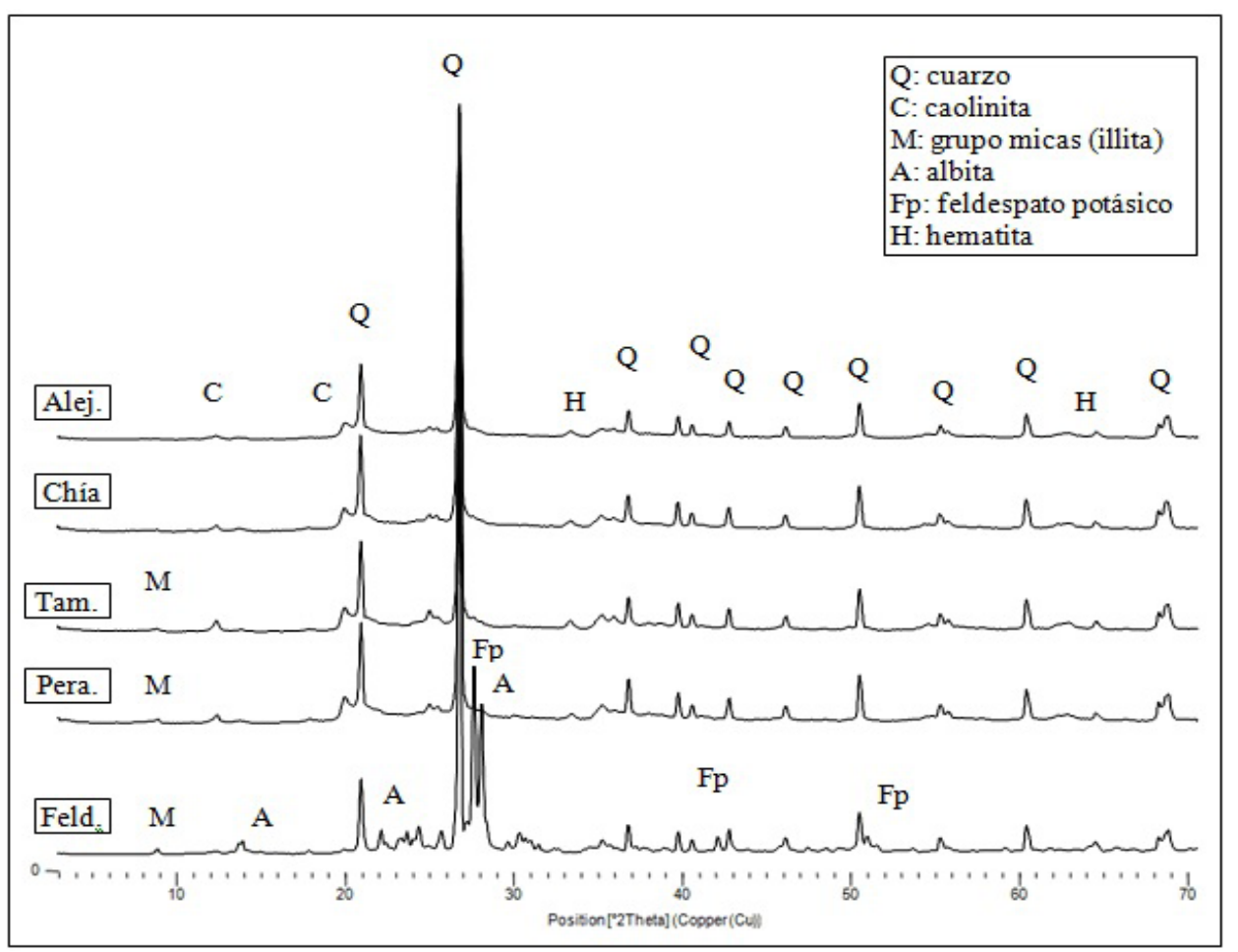

Fig. 1. DRX de las materias primas empleadas

La información arrojada por el analisis de DRX muestra que el mineral predominante en los materiales arcillosos de la zona metropolitana de Cúcuta es el cuarzo libre, seguido por la fracción arcillosa, que es una mezcla de caolinita e illita, principalmente; es probable la presencia de moscovita, pero es difícil diferenciarla de la illita; esta última fase presenta un ligero incremento en las arcillas Támesis y Peracos; se evidencia la presencia de feldespato potásico, hematita y rutilo, como fases minoritarias, que son apenas perceptibles mediante esta técnica.

Los resultados de FRX para todas las muestras presentan bastante similitud; se aprecia un ligero incremento en el contenido de aluminio en las arcillas Támesis y Peracos, probablemente debido a la mayor presencia de fases del grupo de las micas (incluido la illita). Los contenidos de calcio y azufre son bajos, lo cual favorece el uso del material para su aplicación como material de fachada o revestimiento debido a las bajas probabilidades de aparición de eflorescencias. La principal fuente de óxidos fundentes que favorecen la sinterización provienen de la transformación de los minerales del grupo de las micas (potasio principalmente), ya sean la illita o la moscovita a altas temperaturas.
Para el caso del material feldespático, con la información del DRX se pudo establecer que se trata de una mezcla de plagiocasa y feldespato potásico, principalmente; albita y microclina son las fases que más se ajustan en el difractograma; como fases minoritarias se encuentra cuarzo libre y moscovita. La presencia de estas dos fases feldespáticas en las mezclas preparadas podría favorecer la sinterización del proceso, acorde con lo planteado por Avgustinik [10], dando explicación a los resultados de absorción de agua obtenidos; un aporte adicional se podría haber dado por la existencia de moscovita, contribuyendo a una mayor cantidad de óxidos fundentes en la mezcla, mejorando así la sinterización del material cocido; como aspecto desfavorable de la adición de material feldespático se tiene una mayor cantidad de cuarzo libre presente en la mezcla, con lo cual se aumenta el riesgo de fisuras y grietas, debido a los cambios alotrópicos de esta fase cuando se va incrementando la temperatura; por tanto, es recomendable realizar pruebas dilatométricas, a fin de ajustar las curvas de cocción para la fabricación de esta clase de articulos cerámicos. 


\section{B. Caracterización físico-cerámica de las probetas}

1) Contracción en secado y resistencia a la flexión en seco: A las probetas secas se les midió con un calibrador digital su longitud respecto al lado que se relaciona con el ancho del molde de extrusión; los resultados obtenidos se presentan en la Tabla 3. En seguida, las probetas fueron sometidas al ensayo de resistencia mecánica a la flexión, según la norma NTC 4321-4; los resultados obtenidos se pueden apreciar en la misma tabla.

\section{TABLA 3}

CONTRACCIÓN LINEAL Y MÓDULO DE ROTURA (RESISTENCIA A LA FLEXIÓN) DE LAS PROBETAS SECAS

\begin{tabular}{|c|c|c|c|c|}
\hline \multicolumn{5}{|c|}{ Contracción de secado (\%) } \\
\hline Feldespato agregado (\% peso) & $\mathbf{A}$ & $\mathbf{C}$ & $\mathbf{T}$ & P \\
\hline 0,0 & $8,30 \pm 0,42$ & $8,26 \pm 0,64$ & $8,03 \pm 0,54$ & $8,68 \pm 0,43$ \\
\hline 10,0 & $7,78 \pm 0,43$ & $7,75 \pm 0,56$ & $7,24 \pm 0,48$ & $8,00 \pm 0,48$ \\
\hline 20,0 & $7,20 \pm 0,55$ & $7,56 \pm 0,49$ & $6,33 \pm 0,56$ & $7,10 \pm 0,36$ \\
\hline \multicolumn{5}{|c|}{ Módulo de rotura de probetas secas (N/mm $\mathbf{2}$} \\
\hline 10,0 & $6,34 \pm 0,33$ & $4,97 \pm 0,36$ & $9,75 \pm 0,65$ & $6,93 \pm 0,58$ \\
\hline 20,0 & $6,56 \pm 0,38$ & $5,38 \pm 0,43$ & $9,81 \pm 0,76$ & $6,95 \pm 0,43$ \\
\hline & $6,88 \pm 0,50$ & $5,46 \pm 0,47$ & $9,77 \pm 0,72$ & $7,14 \pm 0,61$ \\
\hline
\end{tabular}

A fin de obtener bajos valores de tolerancias dimensionales y alabeo, suele utilizarse materiales desgrasantes (como el caso del feldespato y la arena) [11]; estas variables están directamente relacionadas con el grado de contracción del material seco, por tanto, las mediciones se realizaron sobre este último parámetro. Acorde con lo planteado en la literatura, efectivamente, la adición del material feldespático disminuyó las contracciones de secado en todas las mezclas; para el caso de las mezclas con $20 \%$ de adición de feldespato se obtuvo una reducción de las contracciones entre $8.50 \%$ y $21.20 \%$ respecto a la mezcla patrón; este máximo obtenido fue presentado por la mezcla con presencia de arcilla Támesis.

Al parecer, el mayor tamaño de grano del material feldespático respecto a las arcillas favorece la compactación durante el proceso de moldeo, aportando un ligero aumento de la resistencia mecánica del material seco en todas las mezclas, tal como se puede deducir de los resultados de la Tabla 3 en relación con el módulo de rotura; este hecho resulta de gran interés, ya que podría contribuir a disminuir la cantidad de material no conforme que sale de los secaderos o del que se pierde camino al horno, debido a la manipulación o transporte de ellos.

2) Contracción en cocido, absorción de agua y resistencia a la flexión después de cocción: De forma similar a la realizada con las probetas secas, se realizaron medidas de longitud y cálculo del módulo de rotura de las probetas ya cocidas para los tres tipos de temperatura empleadas $\left(950^{\circ} \mathrm{C}, 1000^{\circ} \mathrm{C} \mathrm{y} 1050^{\circ} \mathrm{C}\right)$; finalmente, a las probetas cocidas se les midió la absorción de agua de acuerdo con lo establecido en la norma NTC 4321-3. Los resultados se pueden apreciar en las Tablas 4, 5 y 6. 


\section{TABLA 4}

\section{RESULTADOS DE CONTRACCIÓN LINEAL A DIFERENTES TEMPERATURAS DE COCCIÓN}

\begin{tabular}{|c|c|c|c|c|}
\hline \multicolumn{5}{|c|}{ Contracción de cocción $\mathbf{9 5 0} \mathbf{}^{\circ} \mathbf{C}(\%)$} \\
\hline Feldespato agregado (\% peso) & $\mathbf{A}$ & $\mathbf{C}$ & $\mathbf{T}$ & $\mathbf{P}$ \\
\hline 0,0 & $0,69 \pm 0,04$ & $1,41 \pm 0,05$ & $0,98 \pm 0,03$ & $1,42 \pm 0,05$ \\
\hline 10,0 & $0,35 \pm 0,03$ & $0,63 \pm 0,01$ & $0,37 \pm 0,02$ & $0,29 \pm 0,01$ \\
\hline 20,0 & $0,22 \pm 0,01$ & $0,59 \pm 0,01$ & $0,19 \pm 0,01$ & $0,25 \pm 0,01$ \\
\hline \multicolumn{5}{|c|}{ Contracción de cocción $\mathbf{1 , 0 0} \mathbf{C} \mathbf{( \% )}$} \\
\hline 0,0 & $1,29 \pm 0,10$ & $2,12 \pm 0,13$ & $1,61 \pm 0,12$ & $1,75 \pm 0,10$ \\
\hline 10,0 & $1,18 \pm 0,05$ & $1,58 \pm 0,07$ & $1,50 \pm 0,13$ & $1,47 \pm 0,07$ \\
\hline 20,0 & $1,01 \pm 0,06$ & $1,48 \pm 0,08$ & $1,18 \pm 0,09$ & $1,14 \pm 0,08$ \\
\hline \multicolumn{5}{|c|}{ Contracción de cocción $\mathbf{1 , 5 0} \mathbf{C} \mathbf{( \% )}$} \\
\hline 0,0 & $3,21 \pm 0,16$ & $4,03 \pm 0,17$ & $2,99 \pm 0,13$ & $3,34 \pm 0,10$ \\
\hline 10,0 & $2,40 \pm 0,09$ & $3,24 \pm 0,17$ & $2,80 \pm 0,16$ & $2,55 \pm 0,10$ \\
\hline 20,0 & $2,29 \pm 0,11$ & $2,78 \pm 0,19$ & $2,58 \pm 0,09$ & $2,51 \pm 0,08$ \\
\hline
\end{tabular}

Las contracciones de cocción también se ven favorecidas por la adición del material feldespático; este hecho lleva a tener unos valores de tolerancia dimensional más bajos y con menor desviación estándar, aspecto de gran interés en este tipo de piezas. A $950^{\circ} \mathrm{C}$, con un $20 \%$ de adición de feldespato, se logró tener una reducción de la contracción de cocción de entre $58 \%$ y $82 \%$; a $1000^{\circ} \mathrm{C}$, para esta misma cantidad adicionada, se alcanza una disminución de entre $21 \%$ y $35 \%$; finalmente, a $1050^{\circ} \mathrm{C}$ se obtiene una reducción de entre $14 \%$ y $31 \%$ respecto a las muestras patrón (sin adición).

\section{TABLA 5}

RESULTADOS DE ABSORCIÓN DE AGUA A DIFERENTES TEMPERATURAS DE COCCIÓN

\begin{tabular}{|c|c|c|c|c|}
\hline \multicolumn{5}{|c|}{ Absorción de agua 950 $\mathbf{C}^{\circ} \mathbf{( \% )}$} \\
\hline Feldespato agregado (\% peso) & $\mathbf{A}$ & $\mathbf{C}$ & $\mathbf{T}$ & $\mathbf{P}$ \\
\hline 0,0 & $12,06 \pm 0,71$ & $12,15 \pm 0,65$ & $10,37 \pm 0,51$ & $12,06 \pm 0,42$ \\
\hline 10,0 & $11,46 \pm 0,52$ & $11,42 \pm 0,79$ & $10,21 \pm 0,73$ & $12,01 \pm 0,76$ \\
\hline 20,0 & $11,15 \pm 0,80$ & $11,17 \pm 0,73$ & $9,85 \pm 0,55$ & $11,36 \pm 0,60$ \\
\hline \multicolumn{5}{|c|}{ Absorción de agua $\mathbf{1 , 0 0}{ }^{\circ} \mathbf{C}(\mathbf{\%})$} \\
\hline 0,0 & $10,94 \pm 0,84$ & $10,18 \pm 0,58$ & $9,76 \pm 0,84$ & $9,64 \pm 0,38$ \\
\hline 10,0 & $10,74 \pm 0,72$ & $9,99 \pm 0,45$ & $9,42 \pm 0,36$ & $9,59 \pm 0,41$ \\
\hline 20,0 & $9,42 \pm 0,69$ & $9,23 \pm 0,62$ & $8,96 \pm 0,49$ & $9,03 \pm 0,22$ \\
\hline \multicolumn{5}{|c|}{ Absorción de agua $\mathbf{1 , 5 0} \mathbf{C} \mathbf{( \% )}$} \\
\hline 0,0 & $8,12 \pm 0,63$ & $7,64 \pm 0,38$ & $7,12 \pm 0,47$ & $8,02 \pm 0,53$ \\
\hline 10,0 & $8,03 \pm 0,55$ & $7,42 \pm 0,46$ & $7,04 \pm 0,52$ & $7,99 \pm 0,45$ \\
\hline 20,0 & $7,86 \pm 0,67$ & $7,03 \pm 0,40$ & $6,85 \pm 0,37$ & $7,36 \pm 0,61$ \\
\hline
\end{tabular}

Los resultados de la prueba de absorción de agua demuestran que ha existido un efecto positivo en la sinterización de los granos a medida que se adiciona feldespato; la absorción se redujo entre $5 \%$ y $8 \%$ a $950^{\circ} \mathrm{C}$; entre $6 \%$ y $14 \%$ a $1000^{\circ} \mathrm{C}$, y entre $3 \%$ y $8 \%$ a $1050^{\circ} \mathrm{C}$, tomando como comparativo las mezclas con $20 \%$ de adición de feldespato. 


\section{TABLA 6}

RESUlTAdOS DE RESISTENCIA A LA FLEXIÓN (MÓDULO DE ROTURA) A DIFERENTES TEMPERATURAS DE COCCIÓN

\begin{tabular}{|c|c|c|c|c|c|c|c|c|}
\hline \multicolumn{9}{|c|}{ Módulo de rotura $950^{\circ} \mathrm{C}\left(\mathrm{N} / \mathrm{mm}^{2}\right)$} \\
\hline $\begin{array}{c}\text { Feldespato } \\
\text { agregado } \\
\text { (\% peso) }\end{array}$ & $\mathbf{A}$ & Des. Est. & $\mathbf{C}$ & Des. Est. & $\mathbf{T}$ & Des. Est. & $\mathbf{P}$ & Des. Est. \\
\hline 0 & 17,91 & 0,91 & 7,44 & 0,56 & 21,14 & 0,84 & 12,99 & 0,63 \\
\hline 10 & 18,95 & 1,12 & 15,21 & 0,97 & 22,36 & 1,36 & 14,25 & 1,01 \\
\hline 20 & 20,36 & 1,10 & 17,28 & 1,00 & 20,71 & 0,98 & 15,86 & 1,32 \\
\hline \multicolumn{9}{|c|}{ Módulo de rotura $1,00^{\circ} \mathrm{C}\left(\mathrm{N} / \mathrm{mm}^{2}\right)$} \\
\hline 0 & 19,42 & 1,30 & 8,28 & 0,91 & 22,06 & 1,07 & 17,66 & 1,20 \\
\hline 10 & 19,99 & 1,05 & 17,43 & 0,94 & 23,32 & 1,45 & 17,75 & 1,54 \\
\hline 20 & 21,74 & 0,98 & 18,33 & 1,22 & 23,91 & 1,23 & 17,98 & 1,32 \\
\hline \multicolumn{9}{|c|}{ Módulo de rotura $1,50^{\circ} \mathrm{C}\left(\mathrm{N} / \mathrm{mm}^{2}\right)$} \\
\hline 0 & 26,55 & 1,42 & 27,27 & 1,89 & 28,45 & 2,14 & 20,78 & 1,75 \\
\hline 10 & 27,45 & 1,37 & 28,56 & 1,46 & 29,71 & 1,26 & 22,36 & 1,57 \\
\hline 20 & 28,92 & 1,25 & 30,05 & 1,35 & 31,52 & 1,19 & 25,73 & 0,98 \\
\hline
\end{tabular}

En relación con la prueba de resistencia a la flexión, se puede apreciar que hay un aumento en el valor del módulo de rotura a medida que se adiciona material feldespático; este resultado está muy ligado a los valores obtenidos en la prueba de absorción de agua, es decir, que dicho efecto se da por una mayor sinterización de los granos, por la presencia fase vítrea (producto de la descomposición de las fases arcillosas) rellenando poros y por la formación de nuevas fases cristalinas que favorecen la resistencia mecánica, muy probablemente se trate de mullita [8-9]. Para esta variable se logró aumentar el módulo de rotura entre $2 \%$ y $132 \%$ a $950^{\circ} \mathrm{C}$, entre $2 \%$ y $121 \%$ a $1000^{\circ} \mathrm{C}$, y entre $9 \%$ y $24 \%$ a $1050^{\circ} \mathrm{C}$, tomando como comparativo las mezclas con $20 \%$ de adición de feldespato.

\section{Conclusiones}

Se evidenció que la adición de material feldespático a las arcillas del área metropolitana de Cúcuta favorece el proceso cerámico con el fin de implementar la producción de piezas cerámicas de gran formato, acorde con los resultados de contracciones de secado y cocción, absorción de agua y resistencia a la flexión (módulo de rotura) obtenidos.
Todas las mezclas preparadas con las diferentes arcillas presentaron la misma tendencia; pero las mezclas preparadas con arcilla Támesis fueron las de mejor comportamiento. Los resultados de contracción presentaron una reducción significativa; en el caso de la etapa de secado se logró disminuir esta variable hasta un máximo de 21,2\% (Támesis) respecto a la muestra patrón, empleando $20 \%$ de adición de feldespato en la pasta cerámica; caso similar se dio para el proceso de cocción, alcanzando un máximo de reducción de $82 \%$ (Peracos) a $950^{\circ} \mathrm{C}$, de $35 \%$ (Peracos) a $1000^{\circ} \mathrm{C}$ y de $31 \%$ (Chía) a $1050{ }^{\circ} \mathrm{C}$, también empleando $20 \%$ de adición de feldespato, $\mathrm{y}$ en relación con sus respectivas mezclas patrón (sin adición).

Las pruebas de absorción de agua y resistencia a la flexión evidencian que la adición del material feldespático favoreció el proceso de sinterización y, probablemente, contribuyó a la formación de fases que aportan resistencia mecánica, acorde con lo expresado en la literatura. La absorción se redujo en un máximo de $8 \%$ (Chía) a $950^{\circ} \mathrm{C}, 14 \%$ (Alejandra) a $1000^{\circ} \mathrm{C}$ y $8,2 \%$ (Peracos) a $1050^{\circ} \mathrm{C}$, tomando como comparativo las mezclas con $20 \%$ de adición de feldespato. En relación con el mejoramiento del módulo de rotura, se logró un incremento máximo de $132 \%$ (mezcla Chía) a $950^{\circ} \mathrm{C}, 121 \%$ (Chía) a $1000^{\circ} \mathrm{C}$ y $24 \%$ (Peracos) a 
$1050{ }^{\circ} \mathrm{C}$, tomando como comparativo las mezclas con $0 \%$ y $20 \%$ de feldespato para ambas pruebas.

\section{Agradecimientos}

Al programa Jóvenes Investigadores e Innovadores de Colciencias, a la empresa GRESCO N.S. S.A.S. y al Centro de Investigación de Materiales Cerámicos (CIMAC), de la Universidad Francisco de Paula Santander, por hacer posible esta investigación. Igualmente, a la empresa Cerámica Italia, por el suministro de las materias primas empleadas en el proyecto.

\section{REFERENCIAS}

[1] J. Sánchez, J. F. Gelves, Y. A. Romero, "Caracterización tecnológica y del talento humano de las empresas fabricantes de cerámica roja ubicadas en el área metropolitana de Cúcuta", Revista Respuestas, año 17, N. ${ }^{\circ} 2$, pp. 71-80, 2012.

[2] D. Torres y J. Sánchez, Análisis del impacto socioeconómico del sector cerámico del Área Metropolitana de Cúcuta a raíz de la problemática actual de las relaciones bilaterales entre Colombia y Venezuela. Universidad Francisco de Paula Santander. Facultad de Ingeniería. Trabajo de grado Ingeniería de Producción Industrial. San José de Cúcuta, 2011.

[3] J. Sánchez, Estrategias para la competitividad del clúster de la cerámica del Área Metropolitana de Cúcuta. Universidad Nacional Experimental del Táchira. Trabajo de grado Maestría en Gerencia de Empresas, Mención Industria. San Cristóbal, Venezuela, 2010.

[4] P. Ramírez, Planteamiento estratégico para el desarrollo de la innovación tecnológica en el sector cerámico del área metropolitana de Cúcuta. Universidad Nacional Experimental del Táchira. Trabajo de grado Maestría en Gerencia de Empresas, Mención Industria, San Cristóbal, Venezuela, 2010.

[5] L. Pineda, Estrategia para la conformación del clúster y plan estratégico para la cadena de cerámica en Cúcuta. Segundo informe. Bogotá D.C, QubitCluster, 2007.

[6] J. Betancur y J. F. Gelves, Diagnóstico de los procesos de secado, cocción y manejo ambiental en las empresas productoras de tableta vidriada de la zona metropolitana de San José de Cúcuta. Universidad Francisco de Paula Santander. Facultad de Ingeniería. Trabajo de grado Ingeniería de producción industrial. San José de Cúcuta. 2006.

[7] J. Sayago y O. Toloza, Estudio de los defectos mayores en la tableta vitrificada de la Ladrillera Casablanca. Universidad Francisco de Paula Santander. Facultad de Ingeniería. Trabajo de grado Ingeniería de Producción Industrial. San José de Cúcuta, 2006.

[8] C. Sánchez, "La mullita y su identificación en materiales cerámicos". Boletín de la Sociedad Española de Cerámica y Vidrio, vol. 5, No. 1, pp. 79-106,1996.

[9] J. Anggono, "Mullite Ceramics: Its Properties, Structure, and Synthesis", Jurnal Teknik Mesin, vol. 7, $\mathrm{N}^{\circ} 1$, pp. 1-10, 2005.

[10] A. I. Avgustinik. Cerâmica. Versión española por Diorki, traductores S.A. Barcelona: Reverté, segunda edición, pp. 125-126, 1983.

[11] P. Díaz del Río P., S. Consuegra y R. Domínguez, "Identificación de una tradición tecnológica cerámica con desgrasante óseo en el neolítico peninsular. Estudio arqueométrico de materiales cerámicos de Madrid (5300-3400 cal AC)", Trabajos de prehistoria, 68, No. 1, pp. 99-122, 2011. 